\title{
Shaping the Future Management of Hepatocellular Carcinoma
}

\author{
Ghassan Abou-Alfa, MD ${ }^{1}$ Massimo Colombo, MD² \\ ${ }^{1}$ Department of Medicine, Memorial Sloan-Kettering Medical Center, \\ Weill Medical College at Cornell University, New York, New York \\ 2 1st Division of Gastroenterology, Fondazione IRCCS Ca' Granda \\ Ospedale Maggiore Policlinico, Università degli Studi di Milano, via F. \\ Sforza 35, Milan, Italy \\ Address for correspondence and reprint requests Ghassan Abou-Alfa, \\ MD, Department of Medicine, Memorial Sloan-Kettering Medical \\ Center, Weill Medical College at Cornell University, 300 East 66th \\ Street, New York, NY 10065 (e-mail: Abou-alg@mskcc.org).
}

Semin Liver Dis 2013;33(S1):S20-S24

\author{
Abstract \\ Keywords \\ - hepatocellular \\ carcinoma \\ - HCC biomarkers \\ - liver cancer \\ - multidisciplinary care \\ - HCC surveillance
}

Several advances in recent years have led to improved surveillance, diagnostic, and treatment options for hepatocellular carcinoma (HCC). Despite these advances, care for this malignancy remains suboptimal, in part because of poor adherence to established guidelines. When diagnosed at an early stage, outcomes for HCC are positive; however, use of appropriate screening techniques and surveillance of at risk patients is still not widely employed. Although a multidisciplinary care team is considered an essential part of successful HCC treatment, fewer than half of the patients with HCC in the United States receive multidisciplinary care. The current suboptimal utilization of potentially curative treatments, including those recommended by treatment guidelines, can be linked to the absence of multidisciplinary care. Additionally, the lack of prognostic and predictive biomarkers for HCC remains challenging, particularly as therapeutic approaches in advanced disease evolve to the use of molecularly targeted agents. Much research is currently focused on the identification of biomarkers for HCC, but improvements in adherence to guidelines for surveillance and appropriate use of available therapies is necessary before care for patients with HCC will improve.
Hepatocellular carcinoma (HCC) is a major global health problem and one of the leading causes of cancer-related death worldwide, ${ }^{1}$ with continued escalation of the clinical and economic burden expected in coming years. Current HCC management and treatment outcomes are dependent on several variables, including the tumor stage, liver function reserve, and patient performance status, and require an individualized multidisciplinary treatment approach. Despite many accomplishments in recent years, several unanswered questions remain and there is a need for further improvement on all fronts of multidisciplinary HCC management. In this review, we will highlight the gaps in implementing recent advances in clinical practice and discuss future perspectives for improving clinical outcomes in HCC.

\section{Improving Multidisciplinary Care at All Levels}

It is widely accepted that a collaborative multidisciplinary approach involving a team of specialists including at least a hepatologist, medical oncologist, surgeon, interventional radiologist, and pathologist, is required for the optimal management of a patient with $\mathrm{HCC}^{2-4}$ This strategy is not being employed to its fullest potential in everyday clinical practice, particularly in the community setting. A recent survey conducted among physicians in the United States indicated that only $\sim 44 \%$ of physicians routinely adopted a multidisciplinary approach, while $38 \%$ discussed their patients at a multidisciplinary meeting only when they were uncertain about management strategy. ${ }^{5} \mathrm{~A}$ similar survey in Europe indicated
Issue Theme Enhancing Clinical Outcomes in Hepatocellular Carcinoma; Guest Editor, Ghassan Abou-Alfa, MD
Copyright @ 2013 by Thieme Medical Publishers, Inc., 333 Seventh Avenue, New York, NY 10001, USA. Tel: +1(212) 584-4662.
DOI http://dx.doi.org/ 10.1055/s-0033-1333633. ISSN 0272-8087. 
that $63 \%$ of physicians applied the multidisciplinary strategy of HCC management. ${ }^{6}$

Despite the endorsement of surveillance measures in at risk patients by both the European and American associations, their effectiveness in clinical practice is suboptimal. ${ }^{2,4}$ In a population-based U.S. study of patients with HCC aged $>65$ years in the Surveillance, Epidemiology, and End-Results (SEER) Registry, only $17 \%$ of patients received regular surveillance and $38 \%$ received inconsistent surveillance in the 3 years prior to HCC diagnosis. ${ }^{7}$ Another analysis showed that even among hepatitis $\mathrm{C}$ virus- (HCV-) infected veterans with cirrhosis who are deemed high risk for development of HCC, regular surveillance was done only in $12.0 \%$ of patients and inconsistent surveillance in $58.5 \%$ of patients. ${ }^{8}$ Along the same lines, global variations in implementation of screening programs for HCC, and therefore treatment patterns for HCC, are well noted. In Japan, active surveillance programs have resulted in a higher frequency of disease presentation at early stages, when curative surgical treatments can be applied. ${ }^{9}$ In Italy, the hospital-based centralized setting enabled the establishment of a large database of patients with chronic liver disease that allows the institution of more rigorous HCC surveillance measures.

Besides these considerations, several other barriers to HCC surveillance might prevent adequate utilization, including physician knowledge gaps regarding guideline recommendations and logistic factors, such as the need for repeat ultrasound every 6 months. A recent analysis performed in the United States showed that patients who were seen by specialists, such as gastroenterologists/hepatologists, were $\sim 4$.5-fold more likely to be screened regularly compared with patients seen in the community setting by primary care physicians. ${ }^{7}$ Thus, it is imperative that more efforts are directed toward community education programs to promote the importance of screening for HCC in patients at risk to diagnose HCC at earlier stages when it has the best chance to be cured.

Although guidelines provide clear recommendations for treatment of HCC, the utilization of potentially curative and noncurative therapies in HCC is perceived to be suboptimal. ${ }^{2}$ Two population-based cohort studies-using the SEER-Medicare-linked database of patients with HCCshowed that less than $30 \%$ of patients aged $\geq 65$ years diagnosedwith HCC received any therapy, ${ }^{10}$ while palliative treatment, including TACE and systemic chemotherapy, was administered in less than $25 \%$ of patients, indicating underutilization of therapy in the United States. ${ }^{10,11}$ Another study underscoring the suboptimal management of HCC reported inappropriate administration of surgical and locoregional therapies to $19.2 \%$ of patients who were deemed unsuitable for such procedures. ${ }^{12}$ In these analyses, lack of access to multidisciplinary care was found to be a major contributor to the observed low utilization of treatment. Therefore, much focus must be directed toward improving comprehensive multidisciplinary strategy at all levels, including the participation and collaboration of academic and community physicians at multidisciplinary HCC meetings and in clinical trials.

\section{Evolving Biomarkers in HCC}

In addition to extensive research of potential molecular targets for therapeutic intervention in HCC, parallel efforts to identify reliable biomarkers for accurate diagnosis in its earliest stages, prediction of recurrence and treatment response, and determination of prognosis might also have a significant effect on improving patient outcomes in the near future.

It is well known that HCC exhibits exceptional genetic complexity and molecular heterogeneity that is characterized by an accumulation of genetic and epigenetic alterations. ${ }^{13}$ However, the key molecular drivers of the progression from chronic liver disease to benign precursor hyperplastic and dysplastic nodules, and finally transformation to HCC remains poorly understood. Diagnosis of early-stage HCC is currently challenging due to a lack of reliable biomarkers. Recently, whole transcriptome analyses have identified biomarkers that differentiate cirrhotic nodules, dysplastic nodules, and early-stage HCC, and potential availability of such biomarkers may increase the diagnostic accuracy of HCC in the future. $^{14-16}$

Although use of surgical resection, local therapies, and liver transplantation in early-stage disease seems well established, it is still unclear which patients would benefit most from each of these modalities. Considering the high recurrence rate after resection, it is unknown if a subset of resected patients might actually benefit more from liver transplantation. It is conceivable that identification of biomarkers for early and late recurrence would aid in better patient selection for these treatment strategies. Recently, provocative genomewide expression profiling data of cirrhotic liver tissue adjacent to tumor tissue revealed a reproducible gene-expression signature that might correlate with late recurrences and with survival. ${ }^{17}$ The investigators of this study proposed that the late recurrences might represent new de novo primary tumors rather than recurrence of the initial tumor, reflecting that exposure to environmental factors, such as viral infections, may predispose hepatocytes to malignant transformation. Recently, a 5-gene signature was found to have prognostic significance in patients with early HCC who underwent surgical resection, and could discriminate good and poor prognosis subgroups, even in patients with tumors $>5 \mathrm{~cm}^{18}$ It is anticipated that such encouraging molecular data could potentially influence therapeutic decisions in the future.

Additionally, utilization of genomic technology has enabled the identification of several subgroups of HCC that differ in terms of genetic and epigenetic alterations, molecular pathways, and clinical features. ${ }^{19-21}$ For example, somatic activating mutations in the oncogene CTNNB1 ( $\beta$-catenin) have been found in $\sim 20-40 \%$ of patients with HCC, and were shown to be associated with chromosomal stability and absence of hepatitis B virus (HBV) infection, although their relation to prognosis remains unclear. ${ }^{22-26}$ In patients with $\mathrm{HBV}$ infection, telomere length may also be correlated to risk and outcome of $\mathrm{HCC}{ }^{27}$ A recent study demonstrated that relative telomere length (determined by a real time 
quantitative polymerase chain reaction) in serum DNA samples of patients with HBV-related HCC was up to $50 \%$ longer than in cancer-free patients with HBV (median, 0.31 vs 0.21 ; $p=0.003) .{ }^{28}$ Mutations in the p53 tumor suppressor gene have also been described; these appear to relate to aflatoxin exposure and HBV infections and are associated with poor prognosis. ${ }^{24}$ Constitutive activation of the Ras/Raf/MAPK pathway plays an important role in hepatocarcinogenesis. Evidence from in vitro studies suggest HCV core proteins may be directly involved in the MAP kinase cascade via constitutive activation of Raf- 1 kinase. $^{29}$ The above data indicate several molecular variants of HCC; however, in contrast to some other cancers where we can individualize therapy based on molecular subtype (e.g., HER2-positive breast cancer, BRAF-mutated melanoma, or EGFR mutation-positive non-small cell lung cancer), ${ }^{30-32}$ no molecular subtype has been identified that can predict response to targeted therapy in HCC. Recent findings from randomized phase II trial indicate that c-Met-expressing HCC may benefit from therapy with the MET inhibitor tivantinib; thus, this biologic subgroup needs to be further explored. ${ }^{33}$ However, the full potential of genetic and molecular research in HCC has been hindered so far, partly by lack of adequate tissue availability. This is certainly changing as the research community continues to recognize the critical need for tissue to help better understand the molecular basis of HCC development and evolution and becomes aware of the importance of including translational research in clinical trial designs.

\section{Concluding Remarks}

Hepatocellular carcinoma is a complex and inherently heterogeneous condition that is confounded by comorbidities. Considerable advances in staging and management of HCC have been made in recent years that have resulted in improved patient outcomes, including survival benefit for advanced HCC. Despite these successes, there is much remaining to be done to "translate efficacy into effectiveness." It is essential that implementation of guideline-recommended surveillance programs is pervasive in both the academic and community settings to diagnose HCC at early stages of disease, and that available treatment options are utilized on an individualized basis. It is of paramount importance that access to integrated multidisciplinary care is established to effectively implement these changes into everyday clinical practice. Recent advances in the molecular genetics and genomics of HCC are expected to favorably influence clinical development of new therapeutics, identification of prognostic and predictive markers, and surrogates of microvascular invasion. Finally, it is increasingly being recognized that changes in the paradigm of clinical trial design and execution are needed to assess and validate these new molecular concepts and novel therapies in future trials.

\section{Acknowledgments}

The author would like to thank Sabitha Muneer, PhD, Bojana Pajk, MD, MSc, Amy Furedy, RN, OCN, and Chelsey
Goins, PhD, for their assistance in writing the manuscript, and Trudy Grenon Stoddert, ELS, for her editorial assistance and assistance preparing the manuscript for publication. Financial support for this supplement was Financial support was provided through an educational grant from Bristol-Myers Squibb Co.

\section{References}

1 Ferlay J, Shin HR, Bray F, Forman D, Mathers C, Parkin DM. GLOBOCAN 2008 v1.2, Cancer Incidence, Mortality and Prevalence Worldwide: IARC CancerBase No. 10 [Internet]. Lyon, FranceInternational Agency for Research on Cancer; 2010. Available at: http:// globocan.iarc.fr/. Accessed June 6, 2012

2 Bruix J, Sherman M. Management of hepatocellular carcinoma: an update. Hepatology 2011;53:1020-1022. Available at: http://www. aasld.org/practiceguidelines/Documents/Bookmarked\&percnt;20 Practice\%20Guidelines/HCCUpdate2010.pdf. Accessed July 3, 2012

3 Omata M, Lesmana LA, Tateishi R, et al. Asian Pacific Association for the Study of the Liver consensus recommendations on hepatocellular carcinoma. Hepatol Int 2010;4(2):439-474

4 European Association For The Study Of The Liver; European Organisation For Research And Treatment Of Cancer. EASL-EORTC clinical practice guidelines: management of hepatocellular carcinoma. J Hepatol 2012;56(4):908-943

5 prIME Oncology. Clinical opinion on the treatment of hepatocellular carcinoma. Survey held at: Symposium on Enhancing Clinical Outcomes in Hepatocellular Carcinoma: A Multidisciplinary Expert Practice; November 3, 2011; San Francisco, CA

6 prIME Oncology. Clinical opinion on the treatment of hepatocellular carcinoma. Survey held at: Symposium on Enhancing Clinical Outcomes in Hepatocellular Carcinoma: A Multidisciplinary Expert Practice; December 3, 2011; Berlin, Germany

7 Davila JA, Morgan RO, Richardson PA, Du XL, McGlynn KA, El-Serag $\mathrm{HB}$. Use of surveillance for hepatocellular carcinoma among patients with cirrhosis in the United States. Hepatology 2010;52 (1):132-141

8 Davila JA, Henderson L, Kramer JR, et al. Utilization of surveillance for hepatocellular carcinoma among hepatitis C virusinfected veterans in the United States. Ann Intern Med 2011; 154(2):85-93

9 Ye S-L, Takayama T, Geschwind J, Marrero JA, Bronowicki J-P. Current approaches to the treatment of early hepatocellular carcinoma. Oncologist 2010;15(Suppl 4):34-41

10 Shah SA, Smith JK, Li Y, Ng SC, Carroll JE, Tseng JF. Underutilization of therapy for hepatocellular carcinoma in the medicare population. Cancer 2011;117(5):1019-1026

11 Davila JA, Duan Z, McGlynn KA, El-Serag HB. Utilization and outcomes of palliative therapy for hepatocellular carcinoma: a population-based study in the United States. J Clin Gastroenterol 2012;46(1):71-77

12 El-Serag HB, Siegel AB, Davila JA, et al. Treatment and outcomes of treating of hepatocellular carcinoma among Medicare recipients in the United States: a population-based study. J Hepatol 2006; 44(1):158-166

13 Buendia MA. Genetics of hepatocellular carcinoma. Semin Cancer Biol 2000;10(3):185-200

14 Kaposi-Novak P, Libbrecht L, Woo HG, et al. Central role of c-Myc during malignant conversion in human hepatocarcinogenesis. Cancer Res 2009;69(7):2775-2782

15 Llovet JM, Chen Y, Wurmbach E, et al. A molecular signature to discriminate dysplastic nodules from early hepatocellular carcinoma in HCV cirrhosis. Gastroenterology 2006;131(6): $1758-1767$

16 Di Tommaso L, Destro A, Fabbris V, et al. Diagnostic accuracy of clathrin heavy chain staining in a marker panel for the diagnosis 
of small hepatocellular carcinoma. Hepatology 2011;53(5): $1549-1557$

17 Hoshida Y, Villanueva A, Kobayashi M, et al. Gene expression in fixed tissues and outcome in hepatocellular carcinoma. N Engl J Med 2008;359(19):1995-2004

18 Nault JC, De Reyniès A, Calderaro J, et al. A molecular signature and algorithm for the diagnosis and prognosis of hepatocellular tumors. Abstract presented at: International Liver Cancer Association Annual Conference; September 2-4, 2011; Hong Kong, China

19 Zucman-Rossi J. Molecular classification of hepatocellular carcinoma. Dig Liver Dis 2010;42(Suppl 3):S235-S241

20 Walther Z, Jain D. Molecular pathology of hepatic neoplasms: classification and clinical significance. Pathol Res Int 2011;2011: 403929

21 Thorgeirsson SS, Grisham JW. Molecular pathogenesis of human hepatocellular carcinoma. Nat Genet 2002;31(4):339-346

22 de La Coste A, Romagnolo B, Billuart P, et al. Somatic mutations of the beta-catenin gene are frequent in mouse and human hepatocellular carcinomas. Proc Natl Acad Sci U S A 1998;95(15): 8847-8851

23 Miyoshi Y, Iwao K, Nagasawa Y, et al. Activation of the beta-catenin gene in primary hepatocellular carcinomas by somatic alterations involving exon 3. Cancer Res 1998;58(12):2524-2527

24 Laurent-Puig P, Legoix P, Bluteau O, et al. Genetic alterations associated with hepatocellular carcinomas define distinct pathways of hepatocarcinogenesis. Gastroenterology 2001;120(7):1763-1773

25 Legoix P, Bluteau O, Bayer J, et al. Beta-catenin mutations in hepatocellular carcinoma correlate with a low rate of loss of heterozygosity. Oncogene 1999;18(27):4044-4046
26 Audard V, Grimber G, Elie C, et al. Cholestasis is a marker for hepatocellular carcinomas displaying beta-catenin mutations. J Pathol 2007;212(3):345-352

27 Liu J, Yang Y, Zhang H, et al. Longer leukocyte telomere length predicts increased risk of hepatitis B virus-related hepatocellular carcinoma: a case-control analysis. Cancer 2011;117(18): 4247-4256

28 Liu HQ An JZ, Liu J, et al. Leukocyte telomere length predicts overall survival in hepatocellular carcinoma treated with transarterial chemoembolization. Carcinogenesis 2012;33(5):1040-1045

29 Giambartolomei S, Covone F, Levrero M, Balsano C. Sustained activation of the Raf/MEK/Erk pathway in response to EGF in stable cell lines expressing the hepatitis $\mathrm{C}$ virus (HCV) core protein. Oncogene 2001;20(20):2606-2610

30 Slamon DJ, Leyland-Jones B, Shak S, et al. Use of chemotherapy plus a monoclonal antibody against HER2 for metastatic breast cancer that overexpresses HER2. N Engl J Med 2001;344(11):783-792

31 Flaherty KT, Puzanov I, Kim KB, et al. Inhibition of mutated, activated BRAF in metastatic melanoma. N Engl J Med 2010; 363(9):809-819

32 Lynch TJ, Bell DW, Sordella R, et al. Activating mutations in the epidermal growth factor receptor underlying responsiveness of non-small-cell lung cancer to gefitinib. N Engl J Med 2004; 350(21):2129-2139

33 Rimassa L, Porta C, Borbath I, et al. Tivantinib (ARQ197) versus placebo in patients (Pts) with hepatocellular carcinoma (HCC) who failed one systemic therapy: results of a randomized controlled phase II trial (RCT). J Clin Oncol 2012;30(Suppl): Abstract 4006 